\title{
On big rip singularities
}

\author{
L.P. Chimento ${ }^{a}$ and Ruth Lazkoz ${ }^{b}$ \\ ${ }^{a}$ Dpto. de Física, Facultad de Ciencias Exactas y Naturales, \\ Universidad de Buenos Aires, Ciudad Universitaria \\ Pabellón I, 1428 Buenos Aires, Argentina \\ chimento@df.uba.ar \\ ${ }^{b}$ Fisika Teorikoa eta Zientziaren Historia Saila, \\ Zientzia eta Teknologiaren Fakultatea, Euskal Herriko Unibertsitatea, \\ 644 Posta Kutxatila, 48080 Bilbao, Spain \\ wtplasar@lg.ehu.es
}

\begin{abstract}
In this comment we discuss big rip singularities occurring in typical phantom models by violation of the weak energy condition. After that, we compare them with future late-time singularities arising in models where the scale factor ends in a constant value and there is no violation of the strong energy condition. In phantom models the equation of state is well defined along the whole evolution, even at the big rip. However, both the pressure and the energy density of the phantom field diverge. In contrast, in the second kind of model the equation of state is not defined at the big rip because the pressure bursts at a finite value of the energy density.
\end{abstract}

\section{Introduction}

Investigations on theories with matter fields that violate the weak energy condition were triggered by the influential paper of Caldwell [1, in which he showed that dark energy of that sort would fit very well the supernova-derived luminosity. These models were dubbed phantom cosmologies, and although phantom cosmologies have been investigated from different perspectives, here 
we will only be concerned with those issues related with analytical properties of the models [2]. Interestingly, it has been shown that in general relativity and some of its generalizations phantom matter might make the universe end up in a kind of singularity [3] characterized by divergences in the scale factor $a$, the Hubble factor $H$ and its time-derivative $\dot{H}$. In other words, the scale factor expands so quickly that the scalar curvature $R$ bursts in the limit $a \rightarrow \infty$, which is reached in a finite amount of proper time [3, 4]. These singularity is commonly called the big rip.

In a recent paper [5], the author suggested that the violation of the weak energy condition would not be necessary for producing a singularity in an expanding universe at a finite late-time. In particular, he showed that a singularity of that sort can be constructed in such a way that the strong weak energy condition is preserved. Although this singularity is different than the usual big rip, both singularities share the same physical attribute of producing a divergent scalar curvature. This is an interesting point because if we enlarge the definition of a big rip by saying it occurs when the scalar curvature diverges, then we will have enriched our understanding about the meaning of a singularity reached at finite time. Hence, it is interesting to clarify the nature of both types of singularities, and we devote this comment to that purpose.

\section{Future late-time singularities}

Let us discuss the two approaches to obtaining big rip singularities. An example of the first one can be obtained when phantom cosmologies are generated by a scalar field with negative kinetic term. There, the energy density and pressure of the field are

$$
\begin{aligned}
\rho_{p h} & =-\frac{1}{2} \dot{\phi}^{2}+V(\phi), \\
p_{p h} & =-\frac{1}{2} \dot{\phi}^{2}-V(\phi),
\end{aligned}
$$

while the Einstein-Klein-Gordon equations read

$$
\begin{gathered}
3 H^{2}=-\frac{1}{2} \dot{\phi}^{2}+V(\phi), \\
\ddot{\phi}+3 H \dot{\phi}-\frac{d V}{d \phi}=0 .
\end{gathered}
$$


For the exponential potential

$$
V=\frac{2\left(6+A^{2}\right)}{A^{4}} e^{-A \phi}
$$

with

$$
\phi=\frac{2}{A} \ln t
$$

the power-law evolutions

$$
a=(-t)^{-2 / A^{2}}
$$

are solutions of the Eqs. (3)-(4). They represent a universe without initial singularity beginning in the remote past with a null scale factor. Thereafter, the scale factor grows monotonically till a future big rip is reached at $t=0$. In our models, $\rho>0$ but the weak energy condition is violated because $\rho+p=-\dot{\phi}^{2}<0$. So, if we associate a perfect fluid with the phantom field, then the barotropic index for the power-law solutions (7)

$$
\gamma_{p h}=-\frac{\dot{\phi}^{2}}{\rho_{p h}}=-\frac{A^{2}}{3},
$$

becomes negative while both the energy density $\rho_{p h}$ and the pressure $p_{p h}$ of the field

$$
\begin{array}{r}
\rho_{p h}=\frac{12}{A^{4} t^{2}} \\
p_{p h}=-\frac{4\left(3+A^{2}\right)}{A^{4} t^{2}},
\end{array}
$$

burst at $t=0$. Note as well that the pressure is negative, as it must, because at the big rip super-acceleration occurs. This kind of solutions were also found by solving the semiclassical Einstein equations for conformally invariant free fields and a conformally coupled massive scalar field in spatially flat Friedmann-Robertson-Walker (FRW) spacetimes with no classical radiation or matter (see Ref. [6]).

In Ref. [5] it was shown that a future finite-time singularity can arise in as FRW expanding universe even when the strong-energy condition $\rho+3 p>0$ holds ${ }^{1}$. This can be done, for instance, inserting a selected scale factor with

\footnotetext{
${ }^{1}$ Recalling that for super-acceleration $p<0$ is required, one may see that a consequence of the preservation of the strong-energy condition is the simultaneous fulfillment of the weak-energy condition.
} 
a future finite-time singularity in the the Einstein equations for a flat FRW space

$$
\begin{gathered}
3 H^{2}=\rho, \\
6 \frac{\ddot{a}}{a}=-(\rho+3 p),
\end{gathered}
$$

we can find the energy density and the pressure satisfying the strong energy condition. In Ref. [5] it was chosen a model given by

$$
a(t)=\left(\frac{t}{t_{s}}\right)^{q}\left(a_{s}-1\right)+1-\left(1-\frac{t}{t_{s}}\right)^{n}
$$

where the scale factor evolves in the interval $0<t<t_{s}$ with $a_{s} \equiv a\left(t_{s}\right)$, $1<n<2$ and $0<q \leq 1$. This model represents a universe beginning at a singularity, where the energy density $\rho$ and the pressure $p$ are divergent while the scale factor behaves as $a(t) \approx\left(a_{s}-1\right)\left(t / t_{s}\right)^{q}$, with $a_{s}>1$. It ends in a big rip at $t=t_{s}$ where the expansion rate $H_{s}$ and energy density $\rho_{s}$ are finite positive quantities but the pressure $p_{s}$ is a positive divergent quantity. This type of big rip singularity is different than that appearing in phantom cosmologies. In fact, the final behavior of the scale factor in this model is driven by the peculiar asymptotic form of the equation of state near $t_{s}$. To see that, it will be interesting to find $p=p(\rho)$, in the limit $t \rightarrow t_{s}$, using that the scale factor is a given function of the cosmological time. To begin with, let us expand the scale factor (13) at late times in powers of $t_{s}-t$. The first correction to the constant $a_{s}$ is given by

$$
a \approx a_{s}+\frac{q\left(1-a_{s}\right)}{t_{s}}\left(t_{s}-t\right)
$$

Inserting this approximate solution in the Einstein equations (11) and (12) we obtain the approximate energy density, expressed in powers of $a_{s}-a$, and the corresponding equation of state

$$
\begin{gathered}
\rho \approx \frac{3 q\left(a_{s}-1\right)}{a_{s}^{2} t_{s}^{2}}\left[q\left(a_{s}-1\right)+2 n\left[\frac{a_{s}-a}{q\left(a_{s}-1\right)}\right]^{n-1}\right], \\
p \approx \frac{6 n(n-1)}{a_{s} t_{s}^{2}}\left[\frac{t_{s}^{2}}{2 n q\left(a_{s}-1\right)}\left[\frac{a_{s}^{2}}{3} \rho-\frac{q^{2}\left(a_{s}-1\right)^{2}}{t_{s}^{2}}\right]\right]^{(n-2) /(n-1)} .
\end{gathered}
$$


Hence, in the limit $a \rightarrow a_{s}$, we have a finite energy density

$$
\rho(t) \rightarrow \rho_{s}=\frac{3 q^{2}\left(a_{s}-1\right)^{2}}{a_{s}^{2} t_{s}^{2}},
$$

and a pressure singularity

$$
p(t) \rightarrow \infty,
$$

which is of a logarithmic or a pole-like singularity depending on whether $(n-2) /(n-1)$ is respectively a real or an integer number. Equivalently, (15) and (16) correspond to a finite expansion rate $H_{s}=\left(\rho_{s} / 3\right)^{1 / 2}$ and a divergent $\dot{H} \rightarrow-\infty$, which means, an infinite acceleration $\ddot{a} \rightarrow-\infty$.

Let us interpret now the peculiar fluid given by (15)-(16) as a perfect fluid with equation of state $p=(\gamma-1) \rho$. In the asymptotic limit $t \rightarrow t_{s}$ the barotropic index becomes

$$
\gamma \approx \frac{2 n(n-1) a_{s}}{3 q^{2}\left(a_{s}-1\right)^{2}}\left[\frac{a_{s}-a}{q\left(a_{s}-1\right)}\right]^{n-2} .
$$

The exotic fluids that lead to future late-time singularities represented by the barotropic indexes (8) and (19) do not share the usual properties of the physical fluids i.e., $1 \leq \gamma \leq 2$. In fact, the former is negative $\gamma_{p h}=-A^{2} / 3$, and the latter diverges asymptotically in the limit $t \rightarrow t_{s}$.

Nevertheless, it must be stressed that the presence of a logarithmic or a pole-like singularity in the equation of state is not a sufficient condition for a big rip singularity to occur. This can be understood by considering a particular example of the the van der Waals fluid [7. Its equation of state is

$$
p=\frac{8 w \rho}{3-\rho}-3 \rho^{2}
$$

with $w$ a constant. Near some finite value $\rho_{0}$ such that $\left|\rho_{0}-\rho_{s}\right|$ is sufficiently small we have $|p| \gg \rho$ so that we may set

$$
p \approx \frac{8 w \rho}{3-\rho}
$$

and

$$
2 \dot{H} \approx-p
$$

that is, we have a pressure dominated regime. Now, let us assume that the model enter this regime when $\rho$ takes a value $\rho_{0}$ slightly smaller (larger) than 
$\rho_{s}$. The singularity will be reached when $3 H^{2}=\rho_{s}$, and this will only be possible if $H$ grows (decreases) with time during this pressure dominated epoch till $\rho$ reaches the value $\rho_{s}$. Clearly, equation (22) tells us that $\rho$ will solely transit between $\rho_{0}$ and $\rho_{s}$ if $w<0$ is negative (positive), and, in particular, for all phantom models $(w<-1)$. We may view the situation as if we had two branches of solutions, depending on the sign of $w$, and the reachability of the big rip depends on which branch is being taken.

The latter example gives us hints for drawing more general conclusions. Let us consider a fluid with an equation of state of the form

$$
p \approx \frac{f(\rho)}{\left|\rho-\rho_{s}\right|^{\alpha}}
$$

where $\alpha>0$ is a real or integer number and such that $f$ is an arbitrary function with definite sign and which remains finite as long as $\rho$ does too. The reason why we choose expression (23) is that not only it includes (21) in the $\rho \rightarrow \rho_{s}$ limit, but also (16). Following an argumentation identical to the previous one, we conclude that the big rip singularity will only be reached if $\operatorname{sign}(\mathrm{f}(\rho))=\operatorname{sign}\left(\rho_{0}-\rho_{\mathrm{s}}\right)$, where as before $\rho_{0}$ stands for the value of $\rho$ when the pressure dominated regime begins.

\section{Conclusions}

Summarizing, we believe it is worth widening the definition of big rip singularities by considering cases in which the divergence only appears in the pressure as those models which preserve the strong or weak energy conditions. Nevertheless, the difference between this new way of viewing big rip singularities and the customary one must always be kept in mind.

We have also shown that in the examples discussed the pressure may diverge due to a logarithmic or a pole-like singularity in the asymptotic equation of state. Along this line we have also shown that the presence of such singularities in the equation of state does not ensure per se that the singularity is reachable, and we have discussed which condition must be met for that to occur. 


\section{Acknowledgments}

L.P.C. is partially funded by the University of Buenos Aires under project X223, and the Consejo Nacional de Investigaciones Científicas y Técnicas. R.L. is supported by the University of the Basque Country through research grant UPV00172.310-14456/2002, by the Spanish Ministry of Science and Technology through research grant BFM2001-0988, and by the Basque Government through fellowship BFI01.412.

\section{References}

[1] R.R. Caldwell, Phys. Lett. B 545, 23 (2002).

[2] S. Nojiri and S. Odintsov, Phys. Lett. B 571, 1 (2003); D.J. Liu and X.Z. Li, Phys. Rev. D 68, 067301 (2003); S. Nojiri and S.D. Odintsov, Phys. Lett. B 562, 147 (2003); P. Singh, M. Sami, and N. Dadhich, Phys. Rev. D 68, 023522 (2003); J.G. Hao and X.Z. Li, Phys. Rev. D 68, 083514 (2003); M.P. Dąbrowski, T. Stachowiak, and M. Szydłowski, Phys. Rev. D 68, 103519 (2003); P.F. González-Díaz, Phys. Lett. B 586, 1 (2004); E. Elizalde and J. Quiroga Hurtado, Mod. Phys. Lett. A 19, 29 (2004); A. Feinstein and S. Jhingan, Mod. Phys. Lett A 19, 457 (2004); X.Z. Li and J.G. Hao, hep-th/0303093; H. Stefancic, astro-ph/0310904;V.B. Johri, astro-ph/0311293 I. Brevik, S. Nojiri, S.D. Odintsov, and L. Vanzo, hep-th/0401073; G. Calcagni hep-ph/0402126; S. Nojiri and S.D. Odintsov, astro-ph/0403622; J.G. Hao and X.Z. Li, astro-ph/0404154.

[3] R.R. Caldwell, M. Kamionkowski, N.N. Weinberg, Phys. Rev. Lett. 91, 071301 (2003); P.F. González-Díaz, Phys. Rev. D 68, 021303 (2003); V. Sahni and Y. Shtanov, J. Cosm. Astro. Phys. 0311, 014 (2003);J.G. Hao and X.Z. Li, astro-ph/0309746, A. Yurov, astro-ph/0305019; M. Sami and A. Toporensky, gr-qc/0312009;M. Bouhmadi-López and J.A. Jiménez Madrid, astro-ph/0404540.

[4] L.P. Chimento and R. Lazkoz, Phys. Rev. Lett. 91 211301, (2003).

[5] J.D. Barrow, Class. Quan. Grav. 21, L79 (2004).

[6] P.R. Anderson Phys.Rev. D 33, 1567 (1986). 
[7] G.M. Kremer, Phys. Rev. D 68, 123507 (2003); gr-qc/0401060. 